\title{
Data on Solar Activity for Science
}

\author{
Ishkov V. ${ }^{1,2}$, Sergeyeva N. ${ }^{2}$, Zabarinskaya L. ${ }^{2}$, Nisilevich M. ${ }^{2}$, Kedrov E. ${ }^{2}$, Krylova T. ${ }^{2}$ \\ ${ }^{1}$ IZMIRAN, Moscow, Troitsk, Russia \\ ${ }^{2}$ GC RAS, Moscow, Russia
}

E mail (vitish@wdcb.ru).

Accepted: 24 June 2019

\begin{abstract}
Solar activity has a huge impact on our planet and surrounding space causing various physical phenomena and processes. Observational and experimental data on solar activity are widely used in basic and applied research. Studying of the phenomena occurring on the Sun and in interplanetary space and their influence on the processes in the outer envelopes and inner shells of the Earth is very important. The data of long-term observations are the most valuable. The World Data Center for Solar-Terrestrial Physics in Moscow has a representative collection of the Sun's observation results obtained by the global network of solar and astronomical observatories and instruments installed on spacecrafts.

The article describes the solar activity data stored in the Center and available on the website in open access. The Center is constantly improving and expanding its information resources; new information technologies providing free convenient access to data are applied. Activities on the preservation and efficient use of historical data, as well as a new approach to the data publication with the assignment of digital object identifier (DOI) convenient for citing data are presented in this article.
\end{abstract}

\section{(C) 2019 BBSCS RN SWS. All rights reserved}

Keywords: solar activity, observational data, World Data Center, free access, digital object identifier (DOI), data citation

\section{Introduction}

Nowadays, research is based on a comprehensive analysis of a large amount of multidisciplinary data and modern computer methods of data processing, analysis and interpretation. The World Data Center for Solar-Terrestrial Physics (WDC for STP) in Moscow provides free access to vast amounts of data, long series of observations, a unique and representative collection of domestic and foreign data on solar-terrestrial and space physics, including the observations of solar activity and the interplanetary magnetic field.

The process of active accumulation of geophysical data began 60 years ago during the International Geophysical Year (IGY) 1957-1958. The IGY is the largest scientific project of the twentieth century, the greatest example of worldwide scientific cooperation in our history. This is a period of concentrated efforts to gain a complete understanding of our planet. Sixty seven countries took part in this project and at least fifteen major international scientific organizations. The observations and experiments were aimed at studying all covers of the globe from the Earth's inner core to the outer layers of the atmosphere bordering on interplanetary space and the influence exerted on them by the Sun. During preparation of the IGY project global observing networks of stations and observatories (geomagnetic, ionospheric, seismological, meteorological, and others) were created to achieve progress in the most promising areas of geophysics.

For the IGY, the essential principle was that the entire volume of data to be obtained during the implementation of the research program should constitute a general fund of knowledge available to all. In 1956 the International Council of Scientific Unions (ICSU) and the World Meteorological Organization proposed to create a system of World Data Centers and agreed it with the governments of several countries. Two IGY Participating Committees, both the USA and the USSR, offered to establish and maintain collections of the whole IGY data in their countries. The offers were accepted and two universal WDCs in the USA and the USSR, known as centers A and B, were created for collection of IGY data on the whole complex of observations. The structures of the WDCs A and B included several centers, one of which was the WDC for Solar-Terrestrial Physics in Moscow. Specialized WDCs $\mathrm{C}$ were also established in a number of countries focused on data from one of the sections of the IGY program. The WDCs A, B, C exchanged data among themselves. The centers have become repositories of materials provided by many IGY participants.
The WDC System ensured accumulation of data, their longterm guaranteed storage and availability for researchers in accordance with specially developed rules formulated in the Guide (CSAGI ..., 1959). Specific types of observations for data collection and forms of data presentation were identified in this document and in its subsequent revisions. Periodically the centers issued catalogues of all data held in their repositories.

After the end of the IGY the WDC System was retained for the collection, long-term storage and dissemination of data obtained by observation networks and during implementation of geophysical projects such as International Year of the Quiet Sun (1964-1965), International Year of the Active Sun (1969-1971), the International Magnetospheric Study (1976-1979), International Solar Maximum Year (1979-1981) and so on. For over 60 years, the results of various international and national geophysical projects and programs, the results of observations at stations and observatories, ships, drifting stations, airplanes, satellites, etc., were collected and stored in the WDCs (Kharin \& Sergeyeva, 2007).

The International Polar Year 2007-2008 showed that the old WDC System cannot fully satisfy current data needs. Since 2009 according to the decision of the ICSU a new international structure - World Data System (WDS), is formed (http://www.icsu-wds.org/) in order to consolidate all accumulated multidisciplinary data in a single structure, to develop methods and technologies for data storage that will ensure the safety of information and access to data for using now and in the distant future. The WDS is based on the 50-year experience of the ICSU WDC System and Federation of Astronomical and Geophysical data-analysis Services.

There is a transition from a multitude of stand-alone centers and services towards a global interoperable distributed data system with developed interconnections between individual disciplinary components, with a broader disciplinary and geographical base and with interdisciplinary data processing applications. The WDS is created as a multidisciplinary structure that guarantees free access to quality data and information and the creation of advanced means of access and data processing. Today the WDS has 115 member organizations including 75 Regular Members (fig. 1).

WDC for STP became a regular member of the ICSU-WDS in 2012. It is guided by the basic principles of the WDS Constitution and supports the WDS data policy (WDS ..., 2015). 
At present, significant amounts of planetary geophysical data obtained in our country and abroad are stored in the repository of the WDC for STP (Sergeyeva et al., 2013). Modern technologies are introduced for data management and dissemination. The Center provides data to institutions, organizations and specialists for basic and applied research and educational institutions without any restrictions and free of charge. The WDC for STP has a website in English and Russian (http://www.wdcb.ru/stp/index.en.html) with information for users, catalogues of data, data descriptions and links to other online resources. Free on-line access to a large amount of data on solar-terrestrial disciplines is implemented on the website.

The repository of the WDC for STP is possessed historical and current results of global observations that include data on the
Earth's magnetic field (records of magnetic field variations, one minute, hourly and annual means values of magnetic field elements and geomagnetic activity indices, data on magnetic storms and pulsations), ionospheric phenomena (results of radio sounding of the ionosphere from the Earth's surface and from satellites, data on the absorption of radio waves by the ionosphere and its structural characteristics, the results of measurements of radio noise) and cosmic rays (data on solar and galactic protons, neutrons, mesons from neutron monitors, meson telescopes and ion chambers). The data on solar activity and interplanetary magnetic field are given at the separate section.

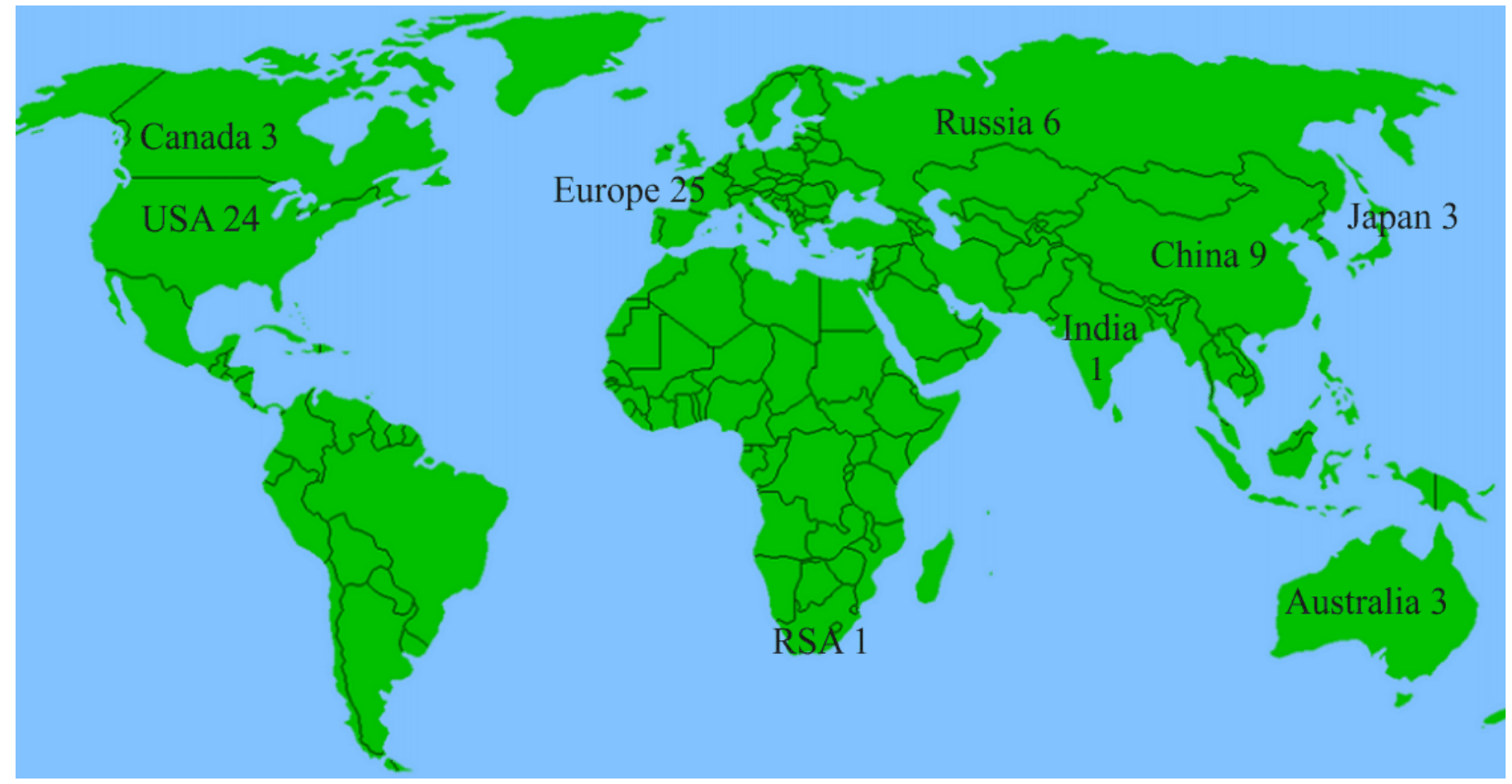

Figure 1. ICSU-WDS Regular Members (April 2019).

\section{Data on Solar Activity in the WDC for STP}

The main data repository of the WDC for STP contains offline historical data (printed or handwritten paper documents) on phenomena that occur on the Sun. These data were obtained from the world network of solar and astronomical observatories and cover the period from 1957 to the beginning of the 1990s. The data include a wide range of routine solar measurements such as sunspot numbers and sunspot groups, their positions and areas, calcium plages, solar magnetic fields, mass emissions from the Sun, prominences and filaments in the $\mathrm{H} \alpha$ line $(656.28 \mathrm{~nm})$. Additionally, synoptic and photographic maps of the Sun, the results of the solar corona optical observations, observations of flares in the $\mathrm{H} \alpha$ line and patrol observations of solar activity, measurements of the total flux of radio emission from the Sun, observations at fixed frequencies of outstanding phenomena on the Sun and radio bursts, radio east-west scans of solar disk, radio spectrograms of solar events are available.

Along with the exchange of observational data, the WDCs exchanged publications containing special data compilations as an important resource for the public, academics and researchers. Among such publications was the "Solar Geophysical Data" distributed monthly by the National Geophysical Data Center (NGDC) of the USA from 1955 to 2009. These reports contain various type collections of observational data on activity of the Sun and its impact on the Earth and information about the main solar events and associated interplanetary, ionospheric and other geophysical effects.
A series of reports "Upper Atmosphere Geophysics" (UAG) published on an irregular basis by the NGDC from 1968 to 1996 is stored in the WDC for STP. These are collections of solar, interplanetary and geophysical data for certain selected time intervals. Among them there are special catalogues on solar active phenomena (flares, coronal holes and emissions of solar filament ejections), collections of brief observational notes with primary processing of individual outstanding events on the Sun, their responses in the interplanetary medium and near-Earth space. All listed is of particular interest to the solar-terrestrial physics community.

The WDC for STP in Moscow also published series entitled "Materials of the World Data Center B" (http://www.wdcb.ru/stp/solar/solar_data.html\#publ2) with data sets on various manifestations of solar activity.

Over time, new methods of observation appeared, the technologies of processing, transmission and storage of information, including the forms of data representation, changed.

With the appearance of instruments with a computer registration, the WDCs began to exchange data in a machinereadable form as data files accompanied by a format description and explanatory text recorded on various media such as magnetic tapes, floppy disks, CD-disks. In the 1980s the unified international formats for data in machine-readable form were adopted to facilitate the exchange and use of information within the scientific community. Data archives in an electronic form and computer databases began to be created. A significant amount of historical data stored in the WDC for STP are in a machinereadable form. A list of these data is available on the Center's 
website (http://www.wdcb.ru/stp/solar/solar_data.html). Data can be obtained on request.

Among the data in machine-readable form stored in the Center it should be noted the obtained by instruments installed on satellites: observations of solar radiation fluxes in the Lymanalpha line from the satellite Explorer during the period 1982-1989, observations in the ultraviolet band from the satellite Explorer-E in 1977-1980, observations in the soft X-ray band (1-8 $\mathrm{A}$ ) from the satellite Solrad (1968-1974) and geostationary satellite GOES. The spacecrafts are equipped with instruments designed to measure total solar radiation - the energy emitted by the Sun at all wavelengths. The measurement of the Earth's radiation above the Earth's atmosphere by a cavity pyrheliometer mounted on the Nimbus-7 satellite (1978-1989) and measurements of the radio emission from the Sun aboard of the orbital station Solar Maximum Mission (1980-1987) are kept by the WDC for STP.

A significant datasets of hourly mean solar wind parameters and interplanetary magnetic field values were gathered from several satellites by Dr. J.H King at the NASA's Goddard Space Flight Center and were provided to the WDC for STP on behalf of the National Space Research Center and WDC A on rockets and satellites.

At the same time with satellite observations ground-based observations in machine-readable form accumulated in the WDCs and are currently available for use:

The values of the coronal solar activity index characterized the total radiation energy of the outermost the Sun atmospheric layer (corona) at the wavelength of $530.3 \mathrm{~nm}$, obtained from the photometric patrol observations at 8 ground stations in 1964-1986.

Data on the relative numbers of sunspots in the international (Zurich) system: maxima and minima, daily, monthly, annual and smoothed values over the entire observation period. Daily reports on the spot number from the Greenwich Observatory (1874-1982), astronomical observatories Rome (1958-1990) and Taipei (19641992). Summaries of sunspot groups from the astronomical observatories Mount Wilson (1967-1989), Rome (1978-1990), Greenwich (1875-2018), Catania (1978-1987).

Data on solar flares from observations in the strongest hydrogen spectral line $\mathrm{H} \alpha$ (1938-1994), a complete list of flares from patrol observations (1955-1994) and a compilation flare index (CFI) calculated for all major flares over the period 19551980.

Measurements of radio noise at ground stations (1960-1989) and spectral radio noise (1967-1989).

The data on calcium plages from solar observatories Hale (1979-1981), Big Bear (1981-1987) and McMath (1942-1979).

Measurements of the solar magnetic field (Sun as a Star), summed over the solar disk (magnetic field intensity in $\mu \mathrm{T}$ ); each value is the weighted average of all measurements during the day. The data of the Crimean Astrophysical Observatory from 1968 to 1976 and the Stanford Solar Observatory from 1975 to 1989.

The density of solar radiation recorded at a frequency of 2800 $\mathrm{MHz}$ by a radio telescope near Ottawa (1947-2018) in the form of tables containing observed, corrected and absolute flux values averaged over daily, monthly and annual intervals. Those data were prepared by Herzberg Institute of Astrophysics, Canada.

Most of the data sets in the modern funds of the WDC for STP are constantly updated, new collections of data in electronic form are formed. Currently, the most frequently used data are published in the "Solar Activity and Interplanetary Medium" section of the WDC for STP w website (http://www.wdcb.ru/stp/solar/solar_activity.html).

One of the most popular parameters (indices) of solar activity is the relative sunspot number (the Wolf number). The WDC for STP website contains the old version of sunspot number data such as daily total sunspot number from 1818 , the average monthly number from 1749 and the average annual number from 1700 . Since July 1st 2015, the original sunspot number data have been replaced by a new entirely revised data series and can be freely downloaded from the website of the WDC for the production, preservation and dissemination of the international sunspot number (WDC-SILSO, Royal Observatory of Belgium, Brussels). In the new version, the influence of sunspot groups is enhanced and heterogeneities in the time series are smoothed (according to authors).

Another solar activity index important for many studies is the flux of the Sun radio emission with a wavelength of $10.7 \mathrm{~cm}$ $(2800 \mathrm{MHz})$, which correlates well with the change in solar activity on the entire visible disk of the Sun. The website provides access to series of daily, monthly and annual values of the radio emission flux measured by the solar radio telescope in three kinds: observed, corrected for changing the Earth-Sun distance and reduced to the mean distance and absolute values. These data are published by the Dominion Radio Astrophysical Observatory (British Columbia, Canada).

Website visitors can look through the table of solar activity cycles which includes the years of the beginning, maximum and minimum of each cycle, the relative sunspot number smoothed over 13 months, the cycle duration and the duration of the increase and decrease branches, as well as the description of the character features of the 24th solar cycle. The catalogues of solar flare events with an X-ray class greater than M1 for the XXIII and XXIV solar cycles are also published here (http://www.wdcb.ru/stp/solar/solar_flare_events.html). The solar flares reflect the energetic of the corresponding magnetic fields, and the behavior of the flare parameters in the 11-year cycle is of considerable interest. The catalogue contains the time parameters for each flare, X-ray class and optical importance of the flare event (observed in the $\mathrm{H} \alpha$ line), integral X-ray flux, coordinates (heliographic latitude, central meridian, Carrington longitude), solar active region number, peak radio flux at selected frequencies, dynamic radio bursts, the accompanying coronal mass ejection (CME), the time and maximum energy of hard $\mathrm{X}$ ray radiation, the maximum flux of solar protons, and the accompanying dynamic phenomena in the optical range.

The WDC for STP website contains electronic versions of six catalogues of solar proton events (SPE) and their energy spectra prepared by teams of specialists in the field of solar-terrestrial physics and edited by Yu.I. Logachev (http://www.wdcb.ru/stp/solar/solar proton events.html). The catalogues include systematized data on the events with proton generation, for which the maximum proton flux with energy Ep > $10 \mathrm{MeV}$ exceeds the value of $1 \mathrm{pfu}$ and contain information about the particle sources and the electromagnetic radiation associated with this event over the available observation range. The catalogues cover the entire period from 1970 to 2008. The integral energy spectrum, tables and graphic images of the proton fluxes (graphic images are only for SPE from 1996 to 2008) at the maximum of the temporal intensity profile are given for each event. Proton flux measurements were taken from the data recorded by various spacecrafts.

Data on the interplanetary magnetic field sector structure include tables containing daily determinations of the polarity direction of the interplanetary magnetic field - from the Sun or to the Sun (sector sign), according to the Stanford University (19471975), and measurements at Vostok and Thule observatories (1974-2010); the table compiled by L. Svalgaard, of the most probable passage times of the field sector boundary, when the polarity of the interplanetary magnetic field reverses, that is, changes to the opposite, and the table and the corresponding graph, containing the azimuthal angles of the interplanetary magnetic field calculated on the basis of the daily average hourly values of the $\mathrm{X}$ and $\mathrm{Y}$ components according to the Goddard Space Flight Center. 


\section{Development of Data Management in the WDC for STP}

In the time since the creation of the WDC System, a significant development of data management technology has occurred from the formation of simple archives, such as libraries, to modern networking technologies of data presentation starting from the publication of data availability catalogs to on-line data and creation of thematic databases and portals. The WDC for STP constantly improves methods of storage, systematization and dissemination of geophysical data and information, providing free network access to them.

Since 1995 the Center's website is a part of the global distributed system of information resources on geophysics. The system of links provides access to data disposed on the websites of other centers, institutes, scientific organizations and researchers.

Nowadays, the project "Preservation of old data" is performed in the WDC for STP with the aim to transfer data from paper medium to electronic form, to increase data sets in electronic form, to prevent the loss of valuable historical data, and, lastly, to provide free on-line access to them for more efficient use.

The second important project entitled "Earth Science DataBase" (ESDB) is in progress with the purpose of creating a modern system for registration and publication of geophysical data with assignment of Digital Object Identifier (DOI) The attitude of the international community towards the data, their distribution, citation and documentation is changed significantly.
Obtaining new data and constant updating of data is considered as a full-fledged scientific research. Consequently scientific data are considered now as valuable results of research and should be cited in the same way as other research information sources: journals, articles, books (Lukyanova, 2013). The DOI system is actively extended to observational science data, including the results of solar and geophysical observations. Each data set with the assigned DOI becomes more accessible for searching, identifying and citing.

Data registration and publication with the assignment of DOI is performed in the WDC for STP through the Crossref agency (Astapenkova et al., 2016). The central repository of the ESDB (http://esdb.wdcb.ru/) contains the metadata for all registered databases and datasets, including complete data descriptions, information about authors, producers, providers and publishers and other information that may be useful to users. For each registered object a landing page is created with complete information on the database/dataset including the URL for data downloading and the citation sample.

For example, individual DOIs were assigned to the entire database of six catalogs of SPEs and to each catalog (dataset) separately. The landing pages were created for the database and each catalog (fig. 2). These solar database and datasets published on the WDC for STP website are available at the address of URL for data downloading pointed on the corresponding landing page (Ishkov, Zabarinskaya, Sergeeva, 2017a).

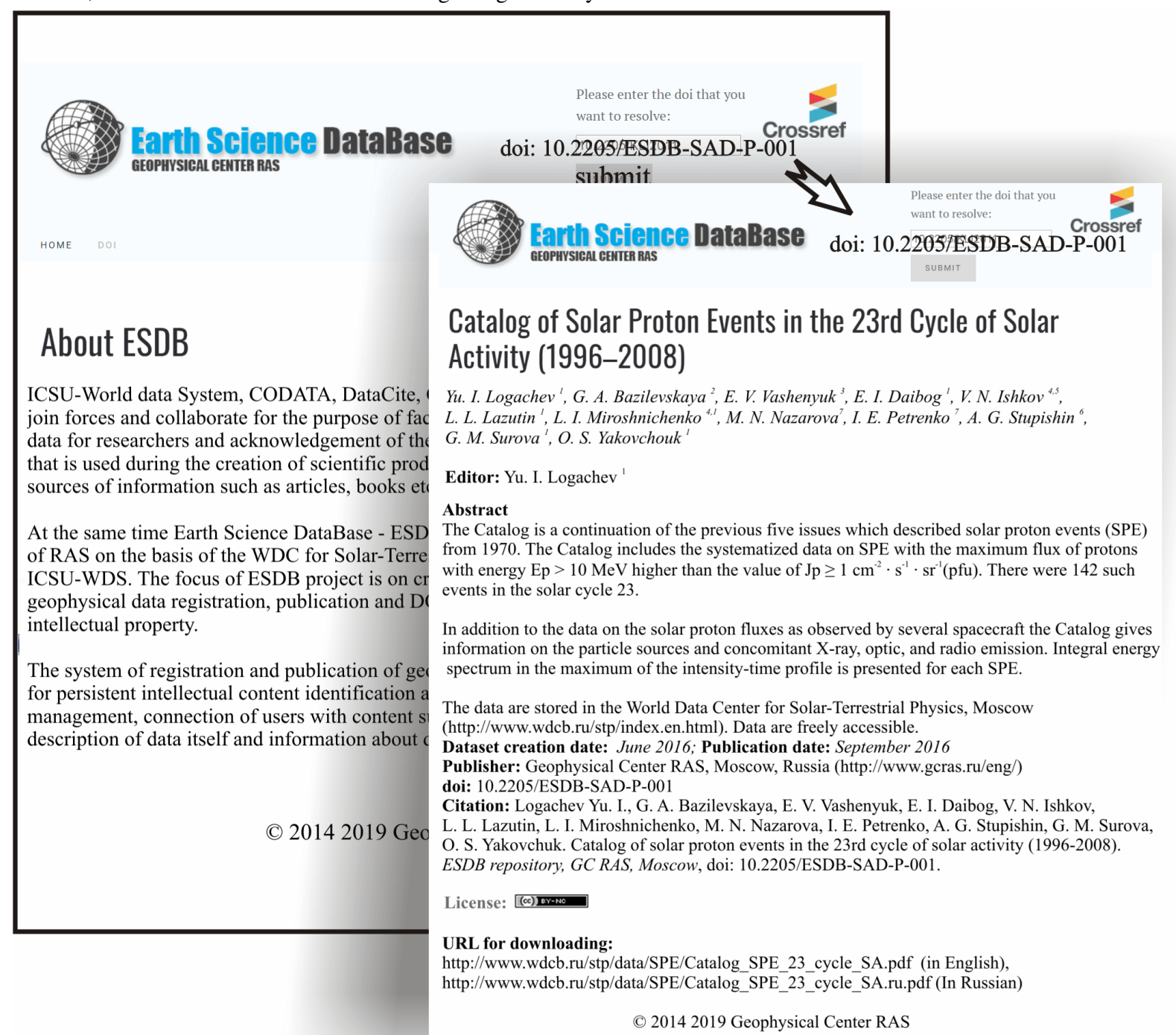

Figure 2. Webpage of the ESDB central repository and the landing page for the Catalog of SPE (Logachev et al., 2016) with DOI used to cite this data. 
These catalogs contain a large amount of information about SPEs. For the convenience of working with such data the modern methods of data organization and presentation are used. The catalog (Logachev et al., 2016) contained data for 142 proton events that occurred in the 23rd solar cycle (1996-2008) is an example of applying a new way of arranging information (Ishkov, Zabarinskaya, Sergeeva, 2017b). A system of links makes possible to switch between individual parts of the catalog and quickly search for the events and their characteristics.

\section{Conclusions}

The World Data Center for Solar-Terrestrial Physics preserving historical data sets, supplementing them with new results of observations, improving data management system, aims to provide scientists with simple and convenient access to updated historical and modern datasets to increase the value of scientific data, to create conditions for their more full and repeated use.

\section{Acknowledgment}

This work was conducted in the framework of budgetary funding of the Geophysical Center of RAS, adopted by the Ministry of Science and Higher Education of the Russian Federation.

This work employed facilities and data provided by the Shared Research Facility "Analytical Geomagnetic Data Center" of the Geophysical Center of RAS (http://ckp.gcras.ru/).

\section{References}

Astapenkova, A.A., Kedrov, E.O., Nechitailenko, V.A., Nisilevich, M.V., Sergeyeva, N.A., Zabarinskaya, L.P.: 2016, Geoinformatics Research Papers. 4, BS4002. DOI:10.2205/2016BS08Sochi.

CSAGI Guide to World Data Centers. 1959, In Annals of the International Geophysical Year, V. 7, 139-374, New York, Pergamon Press.

Ishkov, V.N., Zabarinskaya, L.P., Sergeyeva, N.A.: 2017a, Geomagnetism and Aeronomy. 57, 684-690. DOI: $10.1134 /$ S0016793217060044.

Ishkov, V., Zabarinskaya, L., Sergeeva, N.: 2017b, in Proceedings of Ninth Workshop "Solar Influences on the Magnetosphere, lonosphere and Atmosphere", Sunny Beach, Bulgaria, P. 4653.

Kharin, E. and Sergeyeva, N.: 2007, Earth and Universe. 4. 66-71 (in Russian).

Logachev, Yu.I., Bazilevskaya, G.A., Vashenyuk, E.V., Daibog, E.I., Ishkov, V.N., et al.: 2016. Catalog of Solar Proton Events in

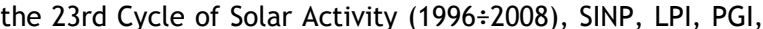
IZMIRAN, GC RAS, SPbU, IAG, Moscow, p. 740. DOI: 10.2205/ESDB-SAD-P-001.

Lukyanova, R.: 2013, Vestnik ONZ RAN. 5, NZ9001. DOI: 10.2205/2013NZ000120 (In Russian).

Sergeyeva, N., Kharin, E., Zabarinskaya, L., Rodnikov, A., Shestopalov, I., Krylova, T., Nisilevich, M.: 2013, Data Science Journal. 12, WDS97-WDS100. DOI: 10.2481/dsj.WDS015.

WDS Data Sharing Principles.: 2015, WDS Scientific Commitee. DOI: $10.5281 /$ zenodo. 34354 